\title{
Hinode magnetic-field observations of solar flares for exploring the energy storage and trigger mechanisms
}

\author{
Toshifumi Shimizu ${ }^{13}$, Satoshi Inoue ${ }^{2}$ and Yusuke Kawabata ${ }^{31}$ \\ ${ }^{1}$ Institute of Space and Astronautical Science, Japan Aerospace Exploration Agency, \\ 3-1-1 Yoshinodai, Chuo-ku, Sagamihara, Kanagawa, 252-5210, Japan \\ email: shimizu@solar.isas.jaxa.jp \\ ${ }^{2}$ Max-Planck Institute for Solar System Research, \\ BT2.E4.120, Justus-von-Liebig-Weg 3 37077, Göttingen, Germany \\ email: inoue@mps.mpg.de \\ ${ }^{3}$ Department of Earth and Planetary Science, The University of Tokyo, \\ 7-3-1 Hongo, Bunkyo-ku, Tokyo 113-0033 Japan \\ email: kawabata.yusuke@ac.jaxa.jp
}

\begin{abstract}
The spectro-polarimeter in the Hinode Solar Optical Telescope (SOT) is one of the powerful instruments for the most accurate measurements of vector magnetic fields on the solar surface. The magnetic field configuration and possible candidates for flare trigger are briefly discussed with some SOT observations of solar flare events, which include X5.4/X1.3 flares on 7 March 2012, X1.2 flare on 7 January 2014 and two M-class flares on 2 February 2014. Especially, using an unique set of the Hinode and SDO data for the X5.4/X1.3 flares on 7 March 2012, we briefly reviewed remarkable properties observed in the spatial distribution of the photospheric magnetic flux, chromospheric flare ribbons, and the 3D coronal magnetic field structure inferred by non-linear force-free field modeling with the Hinode photospheric magnetic field data.
\end{abstract}

Keywords. Sun: flares, Sun: magnetic fields, Sun: photosphere, Sun: chromosphere, Sun: corona

\section{Introduction}

Solar flares abruptly release the free energy stored as a non-potential magnetic field in the corona and may be accompanied by eruptions of the coronal plasma. Magnetic reconnection is considered as a physical process in which the magnetic energy is efficiently converted to kinetic and thermal energies. With the magnetic reconnection, particles in ionized hot plasma are accelerated to non-thermal energy in a short time. Magnetic reconnection is a local process, while the magnetic structure in the corona is destabilized with MHD processes in most of solar flares. The feedback process between magnetic reconnection and MHD destabilization may develop solar flares dynamically and in large scale, resulting in an eruption of magnetic flux ropes.

Before the occurrence of flares, the formation of non-potential magnetic field in the corona is associated with the temporal evolution in the spatial distribution of magnetic flux observed at the solar surface, such as emergence, cancellation, and the horizontal motions of magnetic flux. The location of magnetic reconnection in flaring magnetic structure is difficult to identify directly because of low emission measure at the reconnection region. Thus we are still lack of observational knowledge on the 3D magnetic configuration and physical conditions for leading to flares in the solar atmosphere, especially triggering processes of flares. 


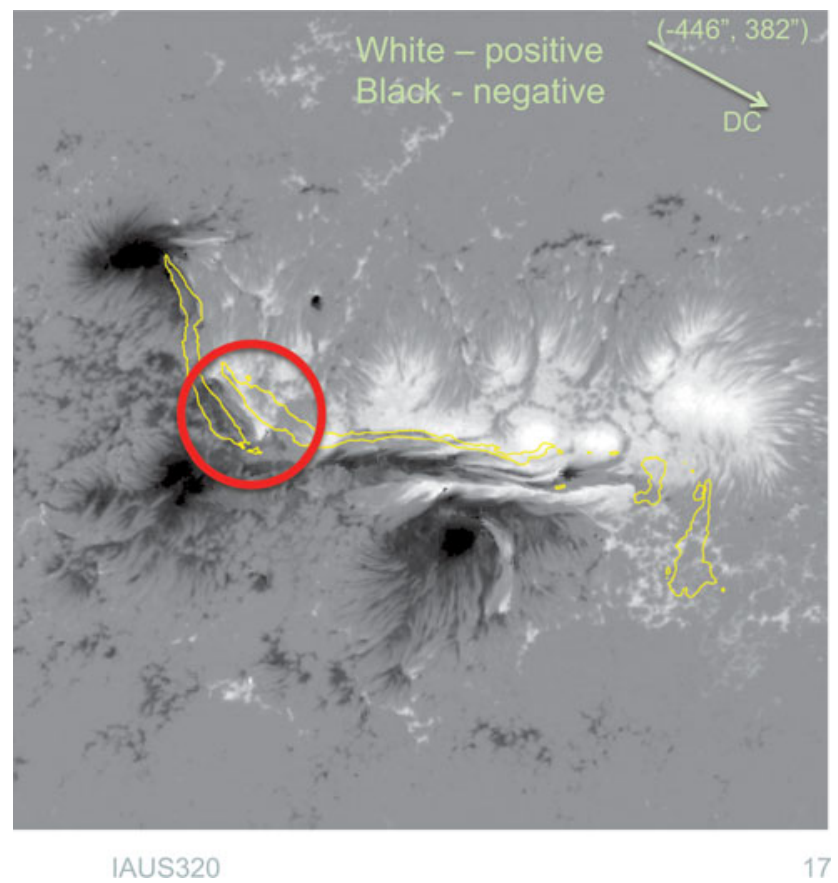

Figure 1. Chromospheric flare ribbons (in yellow) on the spatial distribution of the vertical component of magnetic flux at the photosphere, measured with Hinode Solar Optical Telescope. White and black are positive and negative magnetic polarity, respectively. The red circle indicates the site where a high-speed material flow in the horizontally oriented magnetic field was observed with a small-scale trigger field. The arrow is the direction to the disk center.

Hinode, which was launched in September 2006, has three state-of-art advanced telescopes on board (Kosugi et al. 2007). Of which, Solar Optical Telescope (SOT) is the largest solar telescope on orbit and performs accurate measurements of magnetic flux and its properties at the solar surface with a sub-arcsec spatial resolution, although a narrow field of view (Tsuneta et al. 2008, Suematsu et al. 2008, Shimizu et al. 2008, Ichimoto et al. 2008 ). These accurate measurements of vector magnetic fields at the solar photosphere would help us in exploring how the free energy is stored in the solar atmosphere and how the release of the energy is triggered. This presentation reviewed the magnetic field configuration and possible candidates for flare trigger primarily based on Hinode observations of some large flare events, which included X5.4/X1.3 flares on 7 March 2012, X1.2 flare on 7 January 2014 and two M-class flares on 2 February 2014.

\section{X class flares on 7 March 2012}

The 7 March 2012 events were observed in an active region with delta-type sunspots, showing a strong shear in the entire magnetic system. In Fig. 1, yellow contours give the footpoint location of flaring coronal structure, so-called flare ribbons, at the start of the X5.4 flare. The flare ribbons appeared in a sheared position. For the sheared magnetic structure, the inclusion of a small-scale trigger field was identified near the polarity inversion line with excitation of a high-speed material flow in the horizontally oriented magnetic field formed nearly in parallel to the polarity inversion line (Shimizu et al. 2014). The location of these features is specified by the red circle. The observations 
suggest that gas dynamics at the solar surface play a vital role in leading to the onset of flares.

SDO/AIA movies showed how the coronal magnetic field surrounding the flare site was activated and was evolved toward sudden launch of a plasma eruption. The speed of the plasma ejection was increased in a short time to about $900 \mathrm{~km} / \mathrm{s}$, which is the Alfvenic speed in the corona. The ejected plasma was also observed as a halo CME in the SoHO/LASCO coronagraph field of view. The arrival of protons at the earth was observed within 6-12 hours with the GOES particle monitors.

A couple of the photospheric vector magnetic field maps derived with accurate measurements of Stokes parameters with the spectro-polarimeter (Lites et al. 2013) in SOT were used to infer the 3D coronal magnetic field with non-linear force-free field (NLFFF) modeling (Inoue et al. 2014). The derived results show that low-lying coronal field lines are highly sheared along the polarity inversion line. The spatial distribution of magnetic twist, which is how many turns each field line has from one photospheric footpoint to the other footpoint, showed that high values (mostly in between 0.5 and 1 turns) in magnetic twist are distributed at two elongated areas in parallel to the polarity inversion line. The comparison of the two twist maps, derived with two vector magnetic field maps at about two hours before and after the onset of the flare, shows a variety of changes in the spatial distribution of magnetic twist; The decrease of magnetic twist was primarily observed in the area closer to the polarity inversion line around the site marked by the red circle in Fig. 1, while slight increases were observed at the outer areas apart from the polarity inversion line. This comparison shows how the relaxation took place in the highly sheared magnetic system with the occurrence of the flares. The magnetic twist map was also compared with the temporal evolution in the position of bright flare ribbons, which moved outward from the polarity inversion line as the time went during the flares. The amount of the magnetic twist existing in the location of bright flare ribbons may be in good agreement with the amount of released energy during the flares. The temporal profile of soft X-ray flux showed three peaks during the flares, indicating existence of the three timings in which the energy is abruptly released. The timing of three enhancements in the temporal evolution of the amount of magnetic twist existing in the location of bright flare ribbons is roughly matched with the timing of three soft X-ray peaks, suggesting the importance of magnetic twist for energy storage. Details of the results will be published in a referred journal.

\section{Other flares in January-February 2014}

The 7 January 2014 event is an exceptional event which most scientists would not be able to predict its occurrence. The flare unexpectedly happened apart from the sheared magnetic field region. The M-class flares on 2 February 2014 were observed in the magnetic field configuration, in which four magnetic domains were distributed on the solar surface and an X-shaped point may be formed among the magnetic field lines originating from the four magnetic domains, according to the NLFFF modeling. Details of the results can be found in Kawabata (2016), which should be appeared in a refereed journal later.

\section{Acknowledgements}

Hinode is a Japanese mission developed and launched by ISAS/JAXA, with NAOJ as domestic partner and NASA and STFC (UK) as international partners. It is operated by these agencies in co-operation with ESA and NSC (Norway). We also gratefully acknowledge NASA's Solar Dynamics Observatory and the HMI science team for providing the magnetograms. This work was supported by JSPS KAKENHI Grant Number 23540278 
and $15 \mathrm{H} 05750$. This work is a preview for our coming researches under a new japanese nation-wide project, Project for Solar-Terrestrial Environment Prediction (PSTEP), one of which goals is to make a major improvement in our fundamental knowledge on the energy storage and trigger mechanisms of solar flares toward modeling and predicting dynamical variability in the solar terrestrial environment, so-called space weather in the future.

\section{References}

Ichimoto, K. et al. 2008, Solar Phys., 249, 233

Inoue, S. et al. 2014, Astrophys. J., 780, 101

Kawabata, Y. 2016, Master thesis, The University of Tokyo

Kosugi, T. et al. 2007, Solar Phys., 243, 3

Lites, B. W. et al. 2013, Solar Phys., 283, 579

Shimizu, T. et al. 2008, Solar Phys., 249, 221

Shimizu, T., Lites, B. W., \& Bamba, Y. 2014, PASJ, 66 (SP1), S14

Suematsu, Y. et al. 2008, Solar Phys., 249, 197

Tsuneta, S. et al. 2008, Solar Phys., 249, 167 